\title{
Nurses' Experiences Providing Care for People with Dementia: An Integrative Literature Review
}

\section{Duangrat Monthaisong}

Barry University, Miami Shores, USA

"Corresponding author: Duangrat Monthaisong, PhD candidate, MSN, RN, Barry University, Miami Shores, USA, Tel: +1 3057947449; E-mail: duangrat.monthaisong@mymail.barry.edu

Received date: March 05, 2018; Accepted date: April 19, 2018; Published date: April 23, 2018

Copyright: (C) 2018 Monthaisong D. This is an open-access article distributed under the terms of the Creative Commons Attribution License, which permits unrestricted use, distribution, and reproduction in any medium, provided the original author and source are credited.

\section{Abstract}

Aims and objective: This article seeks to review the experience of registered nurses providing care for dementia patients including theoretical perspectives that have been used to provide care for people with dementia.

Background: Nurses have a vital role in providing special and personalized care for people with dementia and also their family members. However, there is little knowledge about the experiences of nurses providing care for people with dementia in Thailand.

Design: An integrative literature review.

Method: This literature review of 19 articles was undertaken to encapsulate what research has done in the experience of nurses providing care for people with dementia.

Results: Four themes related to the nurses' experiences of providing care for people with dementia included encountering with painful emotions, working under severe conditions, meeting holistic care needs, and experiencing inadequate knowledge. Factors contributing to appropriate care for people with dementia and barriers to meet the optimal care were identified in this article.

Conclusion: Nurses experienced struggling with painful emotions and working under difficult circumstances and there was the need for meeting spiritual care needs of patients. Nurses identified that a lack of knowledge of dementia and the need for improved effective communication when they provided care for a group of these patients and their family members.

Relevance to clinical practice: Nurses seek to understand nurses' experiences of providing care for people with dementia may help them to utilize positive care experiences to practice. Those nurses should consider to some experiences that may be factors to achieve and hinder the optimal care.

Keywords: Nurses' experiences; Providing care; People with dementia

\section{Introduction}

Globally, the structure of the population is changing rapidly from the past. The number of aging population will increase from 900 million in 2015 to 2 billion in 2050 [1]. In Thailand, there are 600,000 people living with dementia, and this number is expected to increase by more than one million by 2030 and triple by 2050 [2]. Dementia is one of the first chronic illnesses to the increased dependency of health and daily activities. In Thailand, as a result of predicted increases in the number of dementia cases and insufficient knowledge of health professionals, the health services will encounter many challenges. There is little understanding of the experiences of nurses providing care for people living with dementia in clinical settings, so there is the need for increased understanding in dementia care. Thus, the concept of interest is the experience of nurses providing care for dementia patients, and this article will guide the future research in Thailand.

\section{Background}

\section{Theoretical perspectives}

Watson's theory of human caring/caring science, Person-Centered Care (PCC) for people with dementia, and spiritual needs as theoretical and conceptual frameworks that have been used to guide the nursing practice such as nursing homes, hospitals, and specialist dementia care units. The theory of human caring has been used to understand nurses' experiences providing care for patients with dementia [3]. Watson [4] describes that human caring is associated with knowledge, interpersonal interaction, values, and compassion and also relates to individual's experience. Person-Centered Care (PCC) has been used to be the clinical guideline in dementia nursing. Holistic care is the principle of person-centered model care to preserve or improve the quality of life for people living with dementia $[5,6]$. Another theoretical perspective that has been used to conduct in dementia nursing care is spirituality as spiritual needs. According to Reed [7] spirituality is defined as the propensity to make meaning 
Page 2 of 6

through a sense of relatedness to dimensions that transcend themselves in such a way that empowers and does not devalue the individual.

\section{Dementia in Thailand}

Since 2002, Thailand has been an aging society with older adults establishing more than 10 percent of the population. Dementia is one of the important chronic diseases. Unfortunately, Thailand does not have national dementia action plans even though the total number of elderly who need someone to help with daily activities is 10 million [8]. Maintaining independence is an essential element of health well-being of the person with dementia [9]. This evidence shows that increased independence of the person with dementia may ensure to positive physical, psychological, spiritual well-being. Nevertheless, there is little knowledge about dementia nursing care in Thailand and programs of dementia care are not entirely developed.

\section{Aim}

The purpose of this article is to review the experience of registered nurses providing care for dementia patients including theoretical perspectives that have been used to provide care for people with dementia. This article also discovers factors contribute to positive and negative nursing care experiences and identifies strategies for managing care for people with dementia.

\section{Methods}

A search of the relevant nursing literature was conducted to find information related to the phenomena of nurses as health providers of people with dementia. A literature search was performed using Internet search engines, such as CINAHL Complete, OVID, MEDLINE Complete, MEDLINE in PubMed, and ProQuest Nursing and Allied Health Source. The author used the keywords in various combinations, including people with dementia, nurses' experiences, nursing care, and providing care. A total of 19 articles that met the criteria were then further formed to identify the core of this integrative literature review.

\section{Results}

All of the studies used a qualitative methodology $(n=19)$ included a reflective journal, phenomenological, ethnographic, grounded theory, descriptive exploratory approaches, an organizational development approach, a pragmatic exploratory approach, and a meta-ethnographic analysis of qualitative studies. A total of 19 articles that met the criteria were studied between 2006 and 2016. Four main themes, each with sub-themes, are summarized in Table 1.

\begin{tabular}{|c|c|}
\hline Themes & Sub-themes \\
\hline \multirow[t]{4}{*}{ Encountering with painful emotions } & Sensing of frustration and exhaustion \\
\hline & Feeling undervalued and meaningless \\
\hline & Feeling vulnerable \\
\hline & Feeling strain \\
\hline \multirow[t]{3}{*}{ Working under severe conditions } & The complexity of caring situations \\
\hline & Work pressure \\
\hline & The difficult struggle to perform person-centered care \\
\hline \multirow[t]{4}{*}{ Meeting holistic care needs } & Sensing and understanding the demented patients' emotional and bodily expressions \\
\hline & Meeting the patient as a person \\
\hline & The need for expressing spiritual care \\
\hline & Being touched by the vulnerability of the demented patient \\
\hline \multirow[t]{2}{*}{ Experiencing inadequate knowledge } & Lack of specific knowledge on dementia care \\
\hline & The need for improved effective communication \\
\hline
\end{tabular}

Table 1: Themes and sub-themes revealed by reduction data analysis.

\section{Encountering difficult emotions}

The theme of struggling with painful emotions was commonly related to nurses' experiences providing care for people with dementia. The multiplicity of feelings has been acknowledged as suffering in providing care for this group of patients because of the progressive nature of the disease and how it psychologically faces with the care recipient. Sensing of frustration and exhaustion, Byers and France [3] found that nine registered nurses reported providing care for people with dementia in the acute care unit was the worst feeling in the world.
Nurses were likely to be consistently negative emotions such as frustrated and exhausted perceptions because they were insufficiently complex needs of people with dementia. All of those healthcare professionals shared their feelings of disappointment and shame. They realized that it was stressful and terrible when they could not take their roles as registered nurses that who are supposed to provide safe care for patients. A study by Hynninen et al. [10] found that lack of nursing' skills led to being exhausted to responsibility for people with dementia in a surgical department, especially exhausting, both physical and mental aspects. 
Feeling undervalued and meaningless, Ostaszkiewicz et al. [11] showed that nurses described a day-to-day work environment in which they were continually at risk of being physically and verbally abused. Working based on unpredictable work environments, insufficient health care professionals and heavy workloads exist, nurses felt undervalued because some patients' family members did not respect them in the way that they deserve. Additionally, Nilsson et al. [12] revealed that emotions evoked when caring for people with dementia such as powerlessness, inadequacy, anger, frustration, stress, fear and being insulted, drained. Losing control of one's feelings was based on descriptions of experiencing the care provided as meaningless to reflect on the care provided.

Feeling vulnerable, Heaslip and Board [13] showed that many nurses felt vulnerability. The staff also shared that dementia was an unpredictable disease and they felt uncertain emotion when they provided care for patients; consequently, they felt threatened and vulnerable. In addition, feeling the strain, Edberg et al. [14] showed that nursing care for people with dementia was mostly a positive experience; however, they still experienced strain from various factors such as environmental factors and the context of caring. Alleviating peoples' suffering and enhancing their quality of life about the encounter with the patient were the factors that caused most difficulties for the nurses in providing care for people with dementia. Because of being unable to provide more care, the nurses did not have the resources, opportunities or abilities to meet the patient's needs; consequently, the experience of strain and frustration increased.

\section{Working under difficult conditions}

The complexity of caring, Ostaszkiewicz et al. [11] indicated that the most common behavioral and psychological symptom of dementia (BPSD) was substantial resistance from residents, which happened when nurses assisted their daily activities. Ostaszkiewicz et al. [11] described that dealing with BPSD was an inevitable part of their dayto-day work. Even though nurses had feeling worn down, stressed, frustrated and hurt, they attempted to not take residents' aggression towards them. Additionally, Hynninen et al. [10] indicated that providing care for people with dementia was specific features of care of an older person with dementia such as responding to physical, psychological as well as social needs and creating a safe environment of care.

Work pressure, McPherson et al. [15] found that nurses and healthcare workers who work in inpatient dementia care wards experienced work stress caused by structural and interpersonal factors such as shift work, the nature of dementia patients, lack of resources, high demand, lack of autonomy, aggression and fear. Nurses and healthcare workers experienced psychological stress such as depression, anxiety, difficulty sleeping or stress in resulting from work pressure. The most staff referred to the need to take a moment and get away from the workplace to relax and deal with difficult feelings and thoughts. Findings indicated that nurses and healthcare workers felt distressed in working on dementia care units as a threat to compassionate care.

The difficult struggle to perform person-centered care, EricsonLidman et al. [6] showed that care providers struggled to perform person-centered care and participants tried to see the person behind the disease and dealt with troublesome in the daily care. Unfortunately, members stated that it was a horrible disease, and it was like they were not themselves anymore. Care providers were in need of clinical supervision, education, and training to be able to provide care for people with dementia properly. Smythe et al. [16] found that staff in a specialist mental health service for person-centered care for people with dementia struggled to define the core skills that they used when providing care for people with dementia. They seemed to be difficult to explain the process, but they felt confident in providing care because it might become automatic and ingrained. Furthermore, staff in a specialist mental health service felt that they had enough knowledge and understanding of person-centered care, but they lacked the time and inadequate staffing to provide care for people with dementia.

\section{Meeting holistic care needs}

Sensing and understanding the demented patients' emotional and bodily expressions, Sellevold et al. [17] revealed that health care providers expressed their understanding of the progress of the dementia disease, and they strived to get through the symptoms of dementia and to reach the person who suffered from this disease. Communicating with and paying attention to the patients were the basis for providing the quality of care such as sitting down, having eye contact, speaking to the patients and focusing on the individual patient. Sensing and understanding the demented patients' emotional and physical expression are ways to meet the high quality of care as a holistic approach [17].

Meeting the patient as a person, Nolan [18] in 2006 found that nurses experienced caring for the older person with dementia were meeting the patient as a person. Nurses desired to care for older adults with dementia as an independent being. They attempted to maximize the individual's potential for independence, and they perceived that a person did not diminish with admission to the acute context. Nurses believed that providing care for this group was the ethical way of being.

The need for expressing spiritual care, Odbehr et al. [19] found that nurses and care workers who provided care for residents with dementia in nursing homes experienced the need for serenity and inner peace, the need to express faith and beliefs, and approaching death. Furthermore, Odbehr et al. [19] revealed that the nurses believed that faith and beliefs were important aspects of the residents' lives, and they realized that the priest as a support in difficult situations they could not handle and did not know how to manage. Additionally, Hynninen et al. [10] found that providing care for people with dementia responded to physical, psychological and social needs. The nursing staff described that response to the physical, psychological and social needs of individuals with dementia and their family members needed more than usual effort and time.

Being touched by the vulnerability of the demented patient, Bryon et al. [20] revealed that being touched by the vulnerability of the demented patient was the heart experience of the nurses. Nurses empathized strongly with the patients and found it to be emotionally difficult to see them in this condition. Providing good care was their guiding principle, which was grounded in being touched by the vulnerability of the patient, so this experience significantly contributed to the nurses professionally and personally.

Striving to provide optimum care, Cowdell in 2010 [21] revealed that nursing staff believed in philosophies of care based on personal beliefs rather than on evidence and they also had great concerns about their capacity to provide care for patients. Nevertheless, nursing staff stated that providing care for people with dementia had to prepare. Most nursing staff described that they gained new knowledge, but it is hard to apply to practice, especially an acute setting. 


\section{Experiencing inadequate knowledge}

Lack of specific knowledge on dementia care, Boersma in 2012 [22] found that there was the need for increased understanding of dementia care. Nurses who worked with a deficit in knowledge and skills might feel a sense of professional failure and frustration when providing care for people with dementia. Increased knowledge and expertise would improve staff competencies and increase confidence with regarding the care of dementia patients. Moreover, Nilsson et al. [23] showed that nursing staff felt inadequate knowledge, lacking specific knowledge of seniors with dementia and their family members. They also stated that they were not having access to person-centered information and this problem contributed to widening the gap between real and ideal care.

The need for improving effective communication, Boersma [22] found that there was no access to shared documentation for colleagues in the acute sector about individual needs, and it relied on the Community Mental Health Team (CMHT) to facilitate this essential information to help a positive experience for the patient. Boersma [22] reflected that in the CMHT, the occupational therapist and physiotherapist remained adamant in their opinions and communication was not effective. Thus, having sufficient communication and overcoming what might have been potentially a difficult situation in dementia care. In addition, Eggers et al. [24] revealed that nursing staff described doing communication was to use different strategies to communicate and understand people with dementia. Nurses especially emphasized the importance of knowing patients' life history, habits and values.

\section{Factors contributing to appropriate care for people with dementia}

Various factors contribute to proper care for people with dementia that include building a relationship between the extended dementia care triad, providing quality time and care in time, attending to the physical and psychosocial needs, and creating a suitable organization, environments, and resources. Scerri et al. [25] found that both health providers and family members identified two other in patients without cognitive impairment and other colleagues that could directly influence this care interaction. Quality dementia care is likely to be based on an extended dementia care triad or triangle of care including the continuous interactions between people with dementia, hospital health providers, family members, and other patients/colleagues. Spending some time out of their busy schedule to get to know their dementia patients better and the provision of quality time referred to the proper relationship between health providers and the patients through the use of appropriate communication skills [23,25]. The most strategy that health providers in the hospital ward setting used in an attempt to meet the patients' psychological needs were the engagement of the meaningful activity of dementia patients. Furthermore, forming relationships between health providers and people with dementia was viewed as a prerequisite to appropriate care [26]. Cowdell in 2010 [21] recommended that health providers needed to listen to physical and psychological needs of the patients and family members and also could learn from their experiences.

Building the suitable organization, environments, and resources, a study of Scerri et al. [25] showed that building the appropriate structure was important in contributing regarding the need for adequate staffing levels. Health providers acknowledged that the need for a homely, safe, clean, and relaxed environment that was suitable for the particular needs of people with dementia. Houghton et al. [26] highlighted the specific conditions were crucial to accommodate people with dementia. Therefore, enhancing organizational factors, physical environment and human resources was fundamental to contributing towards quality care for people with dementia.

\section{Factors hindering appropriate care for people with dementia}

Two main factors that hindered optimal care of people with dementia included nursing staff's skills shortages and distributed responsibility for patients' care [10]. The nursing staff reported that shortage of skills seemed to lead to feel exhausted, and they also stated that providing care for people with dementia was physically and mentally exhausting. Clissett et al. [27] found that most healthcare professionals took actions that seemed to be negative for people with dementia. Importantly, the nursing staff expressed that they lacked competence in managing care for people with dementia and required additional training for caring for patients and their family members [10]. Furthermore, the potential factor of hindering good care for people with dementia is the distributed responsibility for patients' care. Hynninen et al. [10] described that most primary health care staff had the main responsibility for providing care people with dementia, and their competence was for evaluating their care needs; however, physicians retained control of medical decisions and treatment plans. It was also found that medical records were difficult to pass between departments or organizations.

\section{Discussion}

This article has considered the literature related to nurses' experience of providing care for people with dementia and identified several themes and sub-themes, which contribute to both negative and positive experiences. These include encountering with difficult emotions; working under difficult conditions, meeting holistic care needs, and experiencing inadequate knowledge were highly experienced by nursing staff and healthcare providers to occur in hospital settings and nursing homes for people with dementia. Nurses express difficult emotions about providing care for people with dementia include the feeling of frustration and exhaustion, feeling undervalued and meaningless, feeling vulnerable, and feeling the strain. These emotions demonstrate that nursing staffs need to be fully present in knowledge and commitment to their patients and their family members to engage in the caring human transaction to provide safe, holistic quality nursing care. Nevertheless, nursing staff may feel frustrated and exhausted when they are unable to provide proper care for people with dementia. Importantly, this point indicates that negative feelings may affect to nursing staff work that provides care for seniors with dementia. Also, nursing staff may provide care for people with dementia is frustration when they feel dissatisfaction with elements of their role and need dementia-specific training [16].

Furthermore, providing care for people with dementia is likely to work under difficult conditions such as the complexity of caring, work pressure, the difficult struggle to perform person-centered care. The results show that nursing staff still need to understand and clarify the concept of person-centered care that will help them understand and feel confident to provide care for people with dementia. These findings mean that nursing staff is aware of the knowledge that they seek to be responsive to patients' individual needs. Thus, to improve dementia, education and training are important to help nursing staff feel engagement in work and to respond appropriately to people with dementia. The team needs access to training on person-centered care. 
Page 5 of 6

If they have good attitudes and positive feelings, they will deliver quality care to patients.

The findings in this literature also showed that nurses who provided care for people with dementia needed to meet the patients' holistic care needs. These included sensing and understanding the demented patients' emotional and bodily expressions, meeting the patient as a whole person, needing for expressing spiritual care, and being touched by the vulnerability of the demented patients. Caring is an ethical way of being, so nurses are likely to attempt to maximize the people with dementia' potential for independence where the person with dementia is unable to complete an act of living. Many nurses experience spiritual needs not only inter- and intra-personally but also trans-personally. The nurses consider faith-related matters to be important for people with dementia' lives. Indeed, the spiritual needs of individuals with dementia are likely to the rest of the population, aside from the limitations of dementia. In providing care for people with dementia, nurses are likely to meet spiritual needs as providing holistic care.

Based on working with difficult conditions and emotions, nurses encountered many challenges because it was possible that they had inadequate knowledge for dementia care. Many nurses identified that nurses who work with people with dementia needed to be educated and received specialist skills. Because of lack of specific knowledge on dementia care, ineffective communication, and specific features of dementia care, the literature highlights the challenges inherent in providing care for people with dementia in practices such as nursing homes, hospital settings, and facilities of dementia care.

\section{Conclusion}

The review of knowledge regarding the nurse experience of providing care for people with dementia shows a lack of published research focusing on the personal experience of providing care for people with dementia in the nursing lens. Although it may be difficult to gain in-depth information from different approaches and narrow focus of the studies, the majority of the studies have found that nurses tend to have difficult emotions and conditions, lack the knowledge to provide care for people with dementia. However, providing care for people with dementia is likely to provide holistic care approach to patients and their family members. Nurses still need to be educated and trained to encourage their empowerment and confidence to deliver care to patients.

\section{Relevance to clinical practice}

Nurses in clinical settings seeking to understand nurses' experiences of providing care for people with dementia may help them to utilize positive care experiences to practice and should consider to some experiences may be factors to achieve the optimal care.

\section{Recommendations}

Understanding of this phenomenon, future research is needed, and it is important that this event should integrate both quantitative and qualitative studies. Importantly, future research should be identified settings of the phenomenon because different settings may have different environments such as an acute setting, a specialist dementia unit, nursing homes or general wards at hospitals. Also, without a narrow focus of this study may be a barrier to getting in-depth information regarding this phenomenon, so this author believes that narrowing the focus of the particular aspect to one concept (e.g. the experience of holistic care or the end-of-life care) may allow revealing deeper understanding.

\section{References}

1. World Health Organization (WHO) (2017) 10 facts on dementia.

2. Alzheimer's Disease International (2014) Dementia in the Asia Pacific region.

3. Byers DC, France NEM (2008) The lived experience of registered nurses providing care to patients with dementia in the acute care setting: A phenomenological study. Int J Hum Car 2: 44-49.

4. Watson J (2007) Nursing: Human science and human care: A theory of nursing. Sudbury.

5. Stockwell-Smith G, Jones C, Moyle W (2011) You've got to keep account of heads all the time': staff perceptions of caring for people with dementia. J Res Nurs 16: 400-412.

6. Ericson-Lidman E, Larsson LLF, Norberg A (2014) Caring for people with dementia disease (DD) and working in a private not-for-profit residential care facility for people with DD. Scand J Caring Sci 28: 337-346.

7. Reed PG (1992) An emerging paradigm for the investigation of spirituality in nursing. Res Nurs Health 15: 349-357.

8. National Statistics Office of Thailand. (2014). Older persons in Thailand.

9. Moyle W, Fetherstonhaugh D, Greben M, Beattie E (2015) Influencers on the quality of life as reported by people living with dementia in long-term care: a descriptive exploratory approach. BMC Geriatr 15: 50.

10. Hynninen N, Saarnio R, Isola A (2014) The care of older people with dementia in surgical wards from the point of view of the nursing staff and physicians. J Clin Nurs 24: 192-201.

11. Ostaszkiewicz J, Lakhan P, O' Connell B, Hawkins M (2015) On-going challenges responding to behavioural and psychological symptoms of dementia. Int Nurs Rev 62: 506-516.

12. Nilsson A, Rasmussen BH, Edvardsson D (2015) A threat to our integrity - Meanings of providing nursing care for older patients with cognitive impairment in acute care settings. Scand J Caring Sci 30: 48-56.

13. Heaslip V, Board M (2012) Does nurses' vulnerability affect their ability to care? Br J Nurs 21: 912-916.

14. Edberg AK, Bird M, Richards DA, Woods R, Keeley P, et al. (2008) Strain in nursing care of people with dementia: Nurses' experience in Australia, Sweden and United Kingdom. Aging Ment Health 12: 236-243.

15. McPherson S, Hiskey S, Alderson Z (2016) Distress in working on dementia wards - A threat to compassionate care: A grounded theory study. Int J Nurs Stud 53: 95-104.

16. Smythe A, Bentham P, Jenkins C, Oyebode JR (2013) The experiences of staff in a specialist mental health service in relation to the development of skills for the provision of person-centered care for people with dementia. Dementia 14: 184-198.

17. Sellevold GS, Egede-Nissen V, Jakobsen R, Sorlie V (2013) Quality care for persons experiencing dementia: The significance of relational ethics. Nurs Ethics 20: 263-272.

18. Nolan L (2006) Caring connections with older persons with dementia in an acute hospital setting - A hermeneutic interpretation of the staff nurse's experience. Int J Older People Nurs 1: 208-215.

19. Odbehr L, Kvigne K, Hauge S, Danbolt, LJ (2014) Nurses' and care workers' experiences of spiritual needs in residents with dementia in nursing homes: A qualitative study. BMC Nurs 13: 12.

20. Bryon E, Dierckx de Casterlé B, Gastmans C (2012) "Because we see them naked" - Nurses' experiences in caring for hospitalized patients with dementia: Considering artificial nutrition or hydration (ANH). Bioethics 26: 285-295.

21. Cowdell F (2010) Care of older people with dementia in an acute hospital setting. Nurs Stand 24: 42-48. 
Citation: Monthaisong D (2018) Nurses' Experiences Providing Care for People with Dementia: An Integrative Literature Review. J Nurs Care 7: 453. doi:10.4172/2167-1168.1000453

Page 6 of 6

22. Boersma M (2012) A reflection on nurse advocacy for the person experiencing dementia whilst an in-patient in the general and acute sector. Int Pract Dev J 2: 1

23. Nilsson A, Rasmussen BH, Edvardsson D (2015) A threat to our integrity - Meanings of providing nursing care for older patients with cognitive impairment in acute care settings. Scand J Caring Sci 30: 48-56.

24. Eggers T, Ekman SL, Norberg A (2013) Nursing staff's understanding expressions of people with advanced dementia disease. Res Theory Nurs Pract 27: 19-34.

25. Scerri A, Innes A, Scerri C (2015) Discovering what works well: Exploring quality dementia care in hospital wards using an appreciative inquiry approach. J Clin Nurs 24: 1916-1925.
26. Houghton C, Murphy K, Brooker D, Casey D (2016) Healthcare staffs' experiences and perceptions of caring for people with dementia in the acute setting: Qualitative evidence synthesis. Int J Nurs Stud 61: 104-116.

27. Clissett P, Porock D, Harwood RH, Gladman JR (2013) The responses of healthcare professionals to the admission of people with cognitive impairment to acute hospital settings: An observational and interview study. J Clin Nurs 23:1820-1829. 